\title{
Morfologia externa de Thyridia psidii cetoides (Rosenberg \& Talbot) (Lepidoptera, Nymphalidae, Ithomiinae). III. Abdome e apêndices ${ }^{1}$
}

\author{
Jorge Manuel Saraiva Bizarro ${ }^{2}$, Mirna Martins Casagrande ${ }^{2} \&$ Olaf Hermann Hendrik Mielke ${ }^{2}$ \\ ' Contribuição número 1427 do Departamento de Zoologia, Universidade Federal do Paraná. \\ 2 Departamento de Zoologia, Universidade Federal do Paraná. Caixa Postal 19020, 81531-980 Curitiba, Paraná, Brasil. \\ Bolsista CNPq. E-mail: bizarro@xmail.com.br, mibras@ufpr.br, omhesp@ufpr.br
}

\begin{abstract}
External morphology of Thyridia psidii cetoides (Rosenberg \& Talbot). III. Abdomen and appendages (Lepidoptera, Nymphalidae, Ithomiinae). A detailed study of the abdominal external morphology of both sexes of Thyridia psidii cetoides (Rosenberg \& Talbot, 1914) is presented. The material for this research was obtained at the city's plant nursery "Horto Florestal de Curitiba", Paraná, Brazil; mainly by rearing eggs and larvae collected on Cyphomandra betacea (Canavilles) Sendtner, 1845 (Solanaceae). When possible, the results obtained were compared with those already available in the literature concerning other Nymphalidae subfamilies morphology (Brassolinae, Morphinae and Danainae); the most striking feature being the asymmetrical valvae of the male and the length and faint sclerotinization of the third abdominal sternite in both sexes. A resume containing the main morphological differences to other nymphalid subfamillies, found throughout this research concerning head, thorax and abdome, is presented.
\end{abstract}

KEY WORDS. Cyphomandra, comparative morphology, Neotropical.

Finalizando o estudo da morfologia externa do adulto de Thyridia psidii cetoides (Rosenberg \& Talbot, 1914), o presente artigo apresenta aspectos da morfologia externa do abdome de ambos os sexos.

\section{MATERIAL E MÉTODOS}

A metodologia seguida foi idêntica aos dos estudos da cabeça e do tórax (Bizarro et al. 2003a, b), tanto na nomenclatura empregada (MATSUDA 1976), na obtenção e processamento do material, assim como no que se refere às técnicas usadas. As peças foram separadas nos respectivos tagmas - cabeça, tórax e abdome, tendo o cuidado de deixar intacto o segmento visado.

Para o estudo da morfologia do abdome, a estrutura como um todo foi desenhada lateralmente e a genitália retirada posteriormente, abrindo-se o abdome lateralmente pela pleura do sétimo segmento. Foram examinados e comparados machos e fêmeas para detecção de dimorfismo sexual; quando pertinente as estruturas dimórficas foram desenhadas. Salvo indicação oposta, a escala de cada desenho representa $1 \mathrm{~mm}$.

\section{Morfologia do abdome}

O abdome apresenta-se semelhante em ambos os sexos. É composto por dez segmentos, sendo que os dois últimos no macho e os três últimos na fêmea, estão modificados para formarem as respectivas genitálias. Cada segmento com tergo, esterno e pleura, na região central da qual, nos segmentos 1 a 7 estão os espiráculos de forma ovalada (Figs 1-4).

Os tergo e esterno I (Fig. 4) apresentam modificações quando comparados aos outros escleritos, estas modificações estão relacionadas com a articulação tórax-abdome. O tergo tem aspecto de uma bolsa, cuja estreita porção anterior, fortemente esclerotinizada, articula-se com o tórax; o restante é membranoso na parte proximal, e na distal esclerotinizado e projetado sobre o tergo II. Látero e posteriormente, o tergo projeta anteriormente a barra tergo-pleural que contorna dorsalmente o espiráculo I e, na parte distal outra projeção que se une ao esterno I, formando a barra pós-espiracular. $\mathrm{O}$ esterno I é estreito e com uma projeção delgada anterior, que se une à extremidade anterior da barra tergo-pleural, formando a barra pré-espiracular.

Deste modo, o espiráculo I fica completamente contornado pelas barras pré e pós-espiraculares, característica típica de alguns Nymphalidae (Ehrlich 1958b). Neste aspecto, Ithomiinae, Brassolinae e Danainae diferem de Morphinae por esta última não apresentar barra pré-espiracular (BILOTTA 1995), o que não deixa de ser um fato curioso uma vez que EHrLICH (1958b) inclui os Morphinae em Brassolinae, dando por suposta a presença desta estrutura em Morphinae.

O esterno II apresenta, como peculiaridade, a metade do comprimento do tergo equivalente e possui "falsa terminação"; uma região fracamente esclerotinizada, imediatamente anteri-

Revista Brasileira de Zoologia 20 (4): 681-684, dezembro 2003 


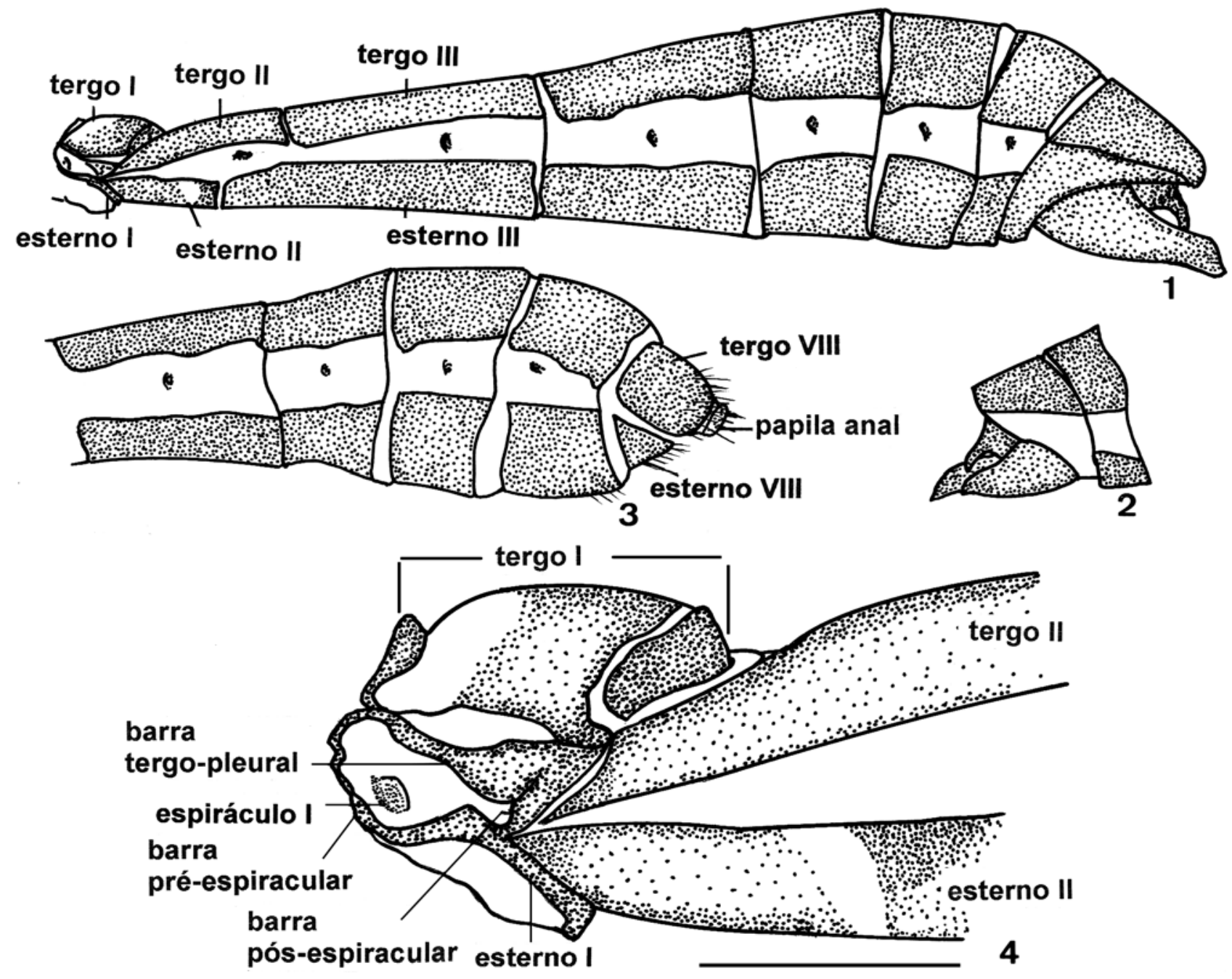

Figuras 1-4. Thyridia psidii cetoides: (1) abdome do macho, vista lateral; (2) fêmea, vista lateral; (3) fêmea, vista ventral; (4) detalhes do primeiro segmento abdominal.

or à extremidade caudal. A combinação deste arranjo estrutural com a forma em bolsa, flexível, do tergo I permite a flexão do abdome em ângulo de pelo menos $90^{\circ}$, facilitando entre outras, a oviposição.

O esterno III também é peculiar, na medida em que é o mais longo dos escleritos abdominais. Os restantes tergos e esternos são simétricos e proporcionais entre si, decrescendo posteriormente em comprimento.

\section{Genitália Masculina (Figs 1-2, 5-7)}

Limitada dorsalmente pelo nono tergo ou tegume, com esclerotinização tão intensa quanto os restantes tergitos e com reforço na região central. Continua-se, sem linha de demarcação nítida, por um prolongamento caudal, o unco, formado pela modificação do décimo tergo, sem processos laterais, se afila progressivamente até formar um gancho e em cuja base, bilateralmente, se encontra um conjunto de pequenas cerdas. Junto à base deste e imediatamente distal às projeções dos braços do tegume, outro par de delgados processos, fracamente esclerotinizados, se encontram na linha mediana formando uma alça limitando externamente a região membranosa onde se abre o orifício anal, esses processos conspícuos constituem o gnato, o X esterno, estrutura comum à todos os Ithomiinae (EHrLich 1958b, Fox 1949, 1967), e residindo neste aspecto a principal diferença da genitália masculina de Ithomiinae (EHrLich 1958b) para com Danainae (Ehrlich 1958a; VANE-Wright \& Ackery 1984), além de Brassolinae (CASAgrande 1979) e espécies de Morphinae (Billota 1995). 


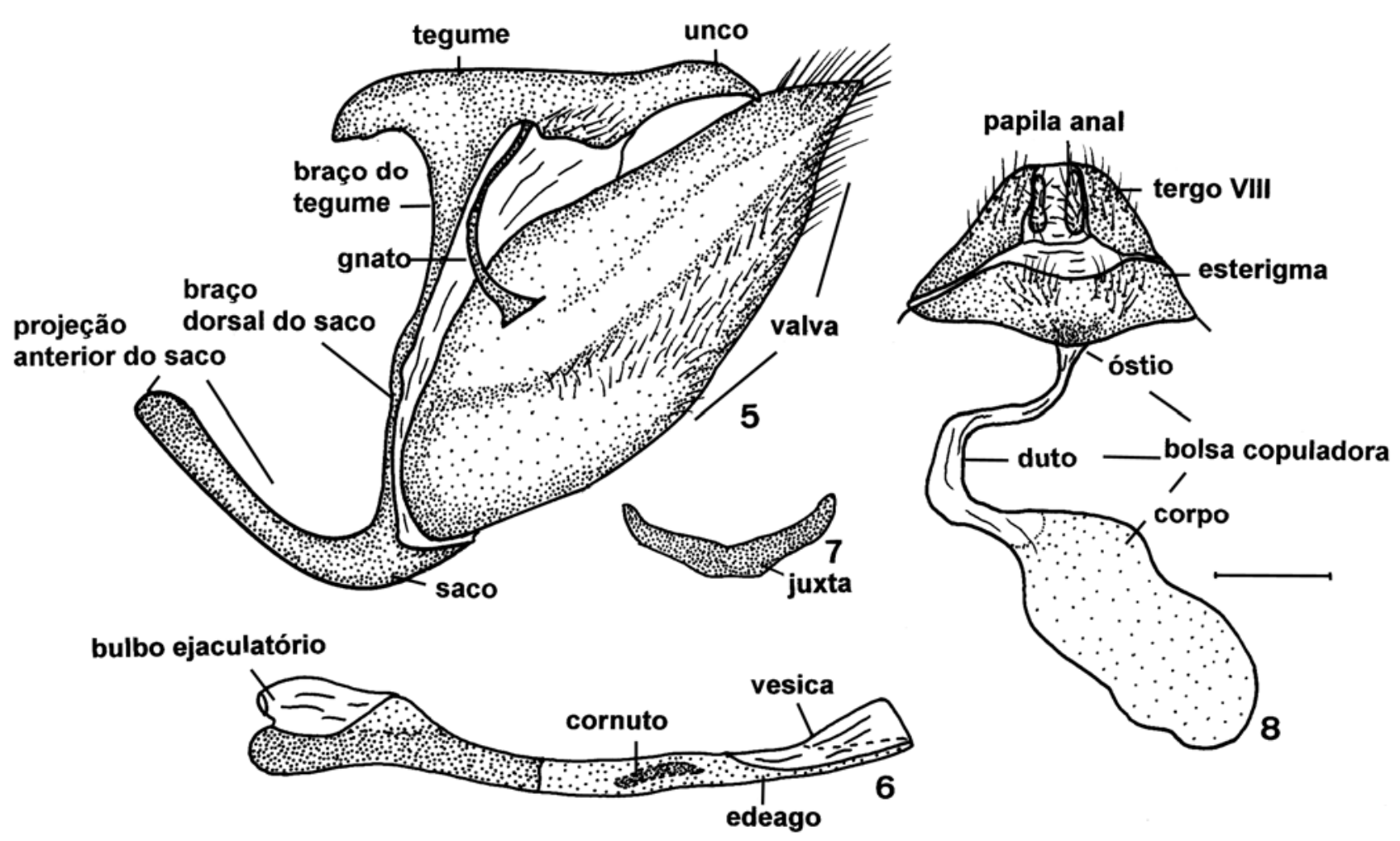

Figuras 5-8. Thyridia psidii cetoides: (5) genitália masculina, vista lateral; (6) pênis, vista lateral; (7) juxta, vista ventral; (8) genitália feminina, vista ventral.

Lateralmente, o tegume emite dois prolongamentos de esclerotinização decrescente, os braços do tegume, que se projetam ventralmente até encontrar os braços dorsais do saco; estrutura esta, ocasionalmente citada na literatura como "vínculo"; um sinônimo de saco (Pierce 1914).

O saco formado pelo nono esterno, possui uma projeção anterior cilíndrica, o prolongamento anterior do saco, e outras látero-dorsais que se unem aos respectivos braços do tegume, os braços dorsais do saco. Não há uma sutura evidente que marque a junção dos braços.

Segundo Niculescu (1978) as valvas seriam os apêndices do décimo segmento, derivados provavelmente da membrana intersegmental do nono e décimo segmentos. Em Thyridia psidii cetoides têm forma e comprimento assimétricos (Fig. 2), podendo a maior delas ser indistintamente a esquerda ou a direita, projetando-se em um pequeno cotovelo na porção dorso-distal, com numerosas cerdas ao longo da face interna, na região distal e bordo ventral, diminuindo de tamanho em sentido ventral.

Abdome fechado distalmente por uma membrana - o diafragma que se estende desde a base das valvas e saco até à base do unco. É dividido transversalmente pelo gnato, com duas aberturas: uma dorsal - o orifício anal, e outra ventral - o forame do edeago, na base e em torno dos quais se encontram um conjunto de minúsculas cerdas. Junto à base do edeago e entre a porção proximal das valvas, está a juxta que dá sustentação ao edeago (Fig. 7).
O pênis é constituído pelo duto e bulbo ejaculatórios, edeago e vesica (Oiticica Filho 1946) (Fig. 6). O bulbo globoso é aberto anteriormente, por onde passa o duto ejaculatório. $\mathrm{O}$ edeago é fino, esclerotinizado, apresentando uma fenda dorsal na extremidade distal, por onde se projeta a vésica, membranosa, com um único cornuto em forma de pena. A presença deste cornuto é outra característica distinta de Thyridia psidii cetoides para com as espécies das outras subfamílias de Nymphalidae já citadas.

\section{Genitália Feminina (Figs 3 e 8)}

Formada pelos oitavo, nono e décimo segmentos abdominais modificados. Os tergos do nono e décimo segmentos abdominais formam as papilas anais, com um par de projeções laterais, dirigidas anteriormente para o interior do abdome, as apófises posteriores, cujas extremidades anteriores são livres, servindo como ponto de inserção dos músculos; nas demais subfamílias comparadas se observam dois pares de apófises, as posteriores e as anteriores, estas últimas do oitavo segmento. As papilas anais, pequenas e devido à estreita membrana intersegmentar, são pouco projetáveis, o que não ocorre em BRASSOLinae (CASAGRande 1979) e Morphinae (Billota 1995), e dispostas lateralmente, ao contrário de Morphinae e Brassolinae (partim), e separadas entre si por uma área membranosa onde se localizam o oviporo e o ânus, sendo este último dorsal ao primeiro. 
O oitavo esterno forma o esterigma, de forma retangular. No meio do esterigma e próximo à margem anterior se abre o óstio da bolsa copuladora que se continua internamente pelo duto da bolsa copuladora, estrutura sinuosa e membranosa, alargada na extremidade proximal, formando o corpo da bolsa, membranosa e saculiforme, sem signos.

A análise final da morfologia do adulto (BIZARro et al. 2003a, b) de Thyridia psidii cetoides, tal como seria de esperar, confirmou outros caracteres entre os disponibilizados na literatura (ACKERY \& VANE-WRIGHT 1984), apontando a subfamília Danainae como aquela cujos caracteres morfológicos mais se assemelham à Ithomiinae, sendo ambas consideradas atualmente como "grupos-irmãos" por esses autores.

Um comparativo entre Thyridia psidii cetoides (Rosemberg \& Talbot, 1914) (Bizarro et al. $2003 \mathrm{a}, \mathrm{b})$ e Danaus plexippus plexippus (Linnaeus, 1758) (EHrLICH 1958a) para os caracteres morfológicos apontados por CASAGRANDE (1979) para Caligo beltrao (Illiger, 1801), e por Billota (1995) para algumas espécies sul-brasileiras de Morphinae é como segue:

Caracteres comuns a Thyridia psidii cetoides e Danaus plexippus plexippus, mas distintos de Caligo beltrao e espécies sulbrasileiras de Morphinae: A) cabeça: sutura transfrontal exígua, entre os soquetes das antenas; faixa transclipeal bem desenvolvida; áreas paraoculares bem desenvolvidas; presença de quetosema reniforme ao longo da sutura temporal; B) tórax: anepisterno II com fortes cerdas; asa anterior com veia $3 \mathrm{~A}$ presente no $1 / 3$ basal; redução do número de tarsômeros da perna protorácica da fêmea; C) abdome: genitália feminina com um único par de apófises.

Caracteres exclusivos de Thyridia psidii cetoides: A) cabeça: côndilos occipitais em forma de aleta semilunar; B) tórax: ausência de pré-episterno e anepisterno I; pelos odoríferos na asa posterior do macho, com inserção sobre a Radial e adjacentes, da base ao fim da célula; C) abdome: esterno III alongado; valvas da genitália masculina conspicuamente assimétricas; gnato em alça semicircular, sustentando o orifício anal

\section{REFERÊNCIAS BIBLIOGRÁFICAS}

ACKeRY, P.R. \& R.I. VANe-Wright. 1984. Milkweed butterflies: their cladistics and biology. Ithaca, Comstock Publishing
Associates, Cornell University Press, IX $+425 p$.

BiLotTa, I.G. 1995. Morfologia comparada do abdome das espécies sulbrasileiras de Morphinae (Lepidoptera, Nymphalidae). Revista Brasileira de Zoologia, Curitiba, 11 (4): 737-748.

Bizarro, J.S.; M.M. Casagrande \& O.H.H. Mielke. 2003a. Morfologia externa de Thyridia psidii cetoides (Rosenberg \& Talbot) (Lepidoptera, Nymphalidae, Ithomiinae). I. Cabeça e Apêndices. Revista Brasileira de Zoologia, Curitiba, 20 (2): 279-284.

. 2003b. Morfologia externa de Thyridia psidii cetoides (Rosenberg \& Talbot) (Lepidoptera, Nymphalidae, Ithomiinae). II. Tórax e Apêndices. Revista Brasileira de Zoologia, Curitiba, 20 (3): 419-425.

Casagrande, M.M. 1979. Sobre Caligo beltrao (Illiger). IV: Morfologia externa do adulto - abdome. (Lepidoptera, Satyridae, Brassolinae). Revista Brasileira de Biologia, Rio de Janeiro, 39 (2): 347-355.

EHrLich, P.R. 1958a. The integumental anatomy of the monarch butterfly Danaus plexippus L. (Lepidoptera - Danaidae). The University of Kansas Science Bulletin, Lawrence, 38 (18): 1315-1349.

. 1958b. The comparative morphology, phylogeny and higher classification of the butterflies. The University of Kansas Science Bulletin, Lawrence, 39: 305-370.

Fox, R.M. 1949. The evolution and systematics of the Ithomiidae (Lepidoptera). University of Pittsburgh Bulletin 45 (8): 36-47.

. 1967. A monograph of the Ithomiidae (Lepidoptera). Part III, the tribe Mechanitini, Fox. Memoirs of the American Entomological Society, Philadelphia, 22: 1-190.

Matsuda, R. 1976. Morphology and Evolution of the Insect Abdomen. Oxford, Pergamon Press, VIII+534p.

Niculescu, E.V. 1978. L'armure génitale chez les Lépidopteres. Bulletin de la Société Entomologique de Mulhouse (Suppl.): 1-98.

Oiticica Filho, J. 1946. Sobre a morphologia do pênis em Lepidoptera. Boletim do Museu Nacional, Nova Série Zoologia, Rio de Janeiro, 50: 1-36.

Pierce, F.N. 1914. The genitalia of the group Geometridae of the Lepidoptera of the British Island. Guildford, City Press, XXIX+84p.

Recebido em 26.IX.2002; aceito em 10.IX.2003. 